\title{
Tratamiento endovascular de aneurisma coronario de rápido crecimiento
}

\author{
Endovascular treatment of fast-growing coronary aneurysm
}

Javier Cóggiola', Cecilia Rindisbacher², Esteban De Giovanni ${ }^{3}$, Leandro Cavana ${ }^{4}$

\section{RESUMEN}

Los aneurismas de arterias coronarias son hallazgos angiográficos inusuales. Describen una dilatación focal de más del $50 \%$ respecto de los segmentos adyacentes normales. La causa más frecuente es la aterosclerosis $(90 \%)$ y sus potenciales complicaciones son la trombosis, ruptura y embolia. Hasta el momento, no hay consenso sobre su manejo; mientras algunos optan por el tratamiento conservador, otros prefieren conductas más agresivas.

Presentamos un caso de aneurisma de arteria descendente anterior en el contexto de SCASEST, tratado exitosamente con angioplastia con stent recubierto con politetrafluoroetileno (PTFE), así como una revisión de la literatura acerca de las opciones terapéuticas disponibles.

Palabras claves: aneurisma coronario; enfermedad coronaria; aterosclerosis, cinecoronariografía; stent recubierto; politetrafluoroetileno.

\section{ABSTRACT}

Coronary artery aneurysms are unusual angiographic findings. Describe a focal dilatation of more than $50 \%$ with respect to normal adjacent segments. The most frequent cause is atherosclerosis (90\%) and its complications related to thrombosis, rupture and embolism. Actually, there is no consensus on its management; while some prefer conservative treatment, others prefer an invasive approach.

We present a case of left anterior descending artery aneurysm presenting as NSTEMI, successfully treated with angioplasty with a stent covered with polytetrafluoroethylene (PTFE), as well as a review of the literature on the therapeutic options available.

Key words: coronary aneurysm; coronary disease; atherosclerosis; cinecoronariography; coated stent; polytetrafluoroethylene.

Revista Argentina de Cardioangiología Intervencionista 2018;9(2):100-102. Doi: 10.30567/RACI/201802/0100-0102

\section{INTRODUCCIÓN}

El aneurisma de la arteria coronaria (AAC) se define como la dilatación localizada (más de 1,5 veces) en comparación con el resto de las arterias coronarias y se clasifican según la forma (saculares o fusiformes), su tamaño y su localización. La incidencia varía del 0,2 al 10\% en la población general. La destrucción de la túnica media es la razón de la formación de AAC. La arteria coronaria derecha es la arteria más comúnmente involucrada, seguida de arteria circunfleja o descendente anterior (diferentes estudios tienen diferentes incidencias) ${ }^{10}$. Se presenta desde formas asintomáticas, como hallazgo incidental en la angiografía, hasta cuadros de dolor precordial y muerte súbita. Presentamos un caso de AAC de rápido crecimiento, que se presenta como SCASEST, y su tratamiento endovascular.

\section{PRESENTACIÓN DEL CASO}

Paciente masculino de 70 años de edad, hipertenso, dislipémico, con antecedentes de angioplastia coronaria con stent convencional en mayo del 2013 en el segmento medio de la arteria descendente anterior con posterior colocación de stent liberador de drogas (zotarolimus) en junio del 2016 por reestenosis intrastent.

1. Médico Cardiólogo. Cardiólogo intervencionista. Médico de planta del Servicio de Hemodinamia del Instituto Privado de Cardiología.

2. Médico Residente. $3^{\circ}$ año de Cardiología, Instituto privado de Cardiología

3. Médico Cardiólogo. Cardiólogo intervencionista. Jefe de Servicio de Hemodinamia del Instituto Privado de Cardiología

4. Médico Cardiólogo. Jefe de Unidad Coronaria del Instituto Privado de Cardiología.

$\triangle$ Correspondencia: Dr. Javier Cóggiola. Rivadavia 3129, S3000FWK Santa Fe, Santa Fe, Rep. Argentina. Tel: (0342) 4561137. Email: javierco16@hotmail.com.

Los autores no declaran conflictos de intereses

Recibido: 14/03/2018|Aceptado: 12/05/2018
Ingresa en septiembre del 2017 a Unidad Coronaria por presentar dolor precordial de carácter opresivo sin irradiación ni síntomas neurovegetativos, sin cambios electrocardiográficos y biomarcadores cardíacos dentro de parámetros normales. Se interpreta como SCASEST, instaurando inmediatamente tratamiento médico específico al cual responde de manera parcial; por ello se decide realizar cinecoronariografía, la cual evidenció una dilatación aneurismática de la arteria descendente anterior a nivel del tercio proximal previa al stent de $7,2 \mathrm{~mm} \times 6,8 \mathrm{~mm}$.

No se toma conducta por cuestiones ajenas al cuerpo médico. Reingresa en noviembre de 2017 por nuevo cuadro de SCASEST y se estudia nuevamente mediante cinecoronariografía, constatándose lesión $70 \%$ ostial de descendente anterior y aumento significativo del tamaño del aneurisma previa-

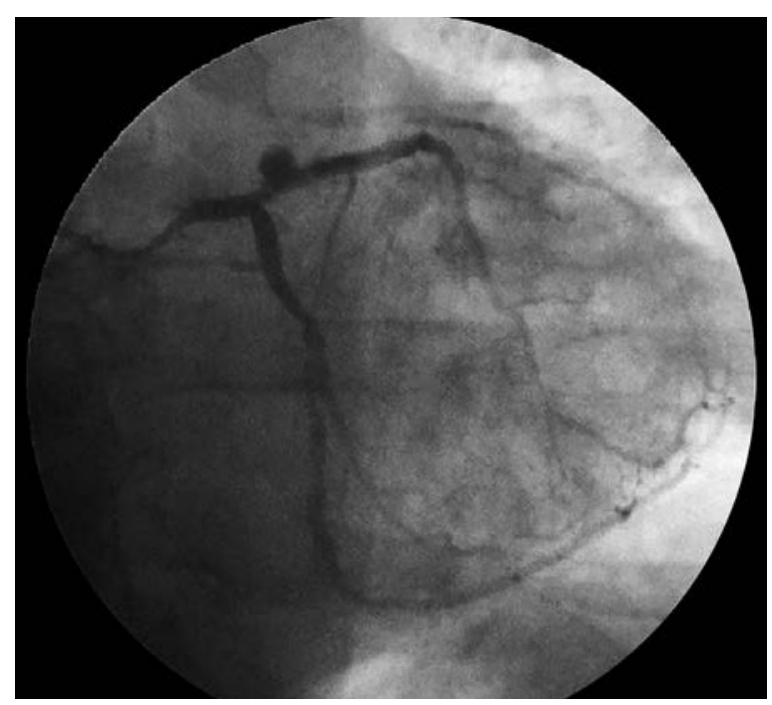

Figura 1. 


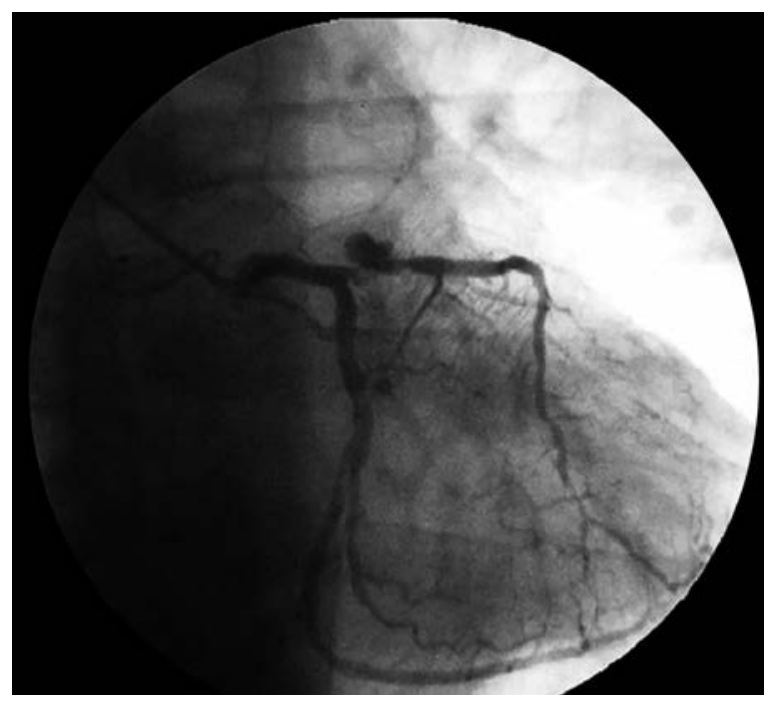

Figura 2.

mente descripto $(10,2 \mathrm{~mm} \times 8,4 \mathrm{~mm})$. Ante dichos resultados, se decide realizar angioplastia con stent recubierto con politetrafluoroetileno.

Mediante punción femoral derecha con introductor $6 \mathrm{Fr}$, se realiza cateterización selectiva de arteria coronaria izquierda con catéter XB 3.5, control angiográfico, donde se constata nuevo aumento significativo del tamaño del aneurisma $(13,9$ $\mathrm{mm} \times 10 \mathrm{~mm}$ ); se realiza pasaje de dos guías 0.014 hacia lecho distal de circunfleja y de descendente anterior; se procede a predilatar con balón $2.0 \times 15 \mathrm{~mm}$ a 12 ATM a nivel de estenosis ostial. Posterior a esto, se posiciona stent cubierto Begraft $3.5 \times 18 \mathrm{~mm}$ que se impacta e implanta a 12 ATM. La angiografía final muestra la arteria permeable con exclusión completa del aneurisma y resolución completa de estenosis en segmento ostial.

El paciente evolucionó favorablemente y sin complicaciones durante la internación. Actualmente concurre a controles con médico de cabecera, manteniéndose asintomático y con prueba funcional negativa para isquemia.

\section{DISCUSIÓN}

Los aneurismas de las arterias coronarias son hallazgos angiográficos inusuales y asintomáticos en su mayoría. Dicha patología fue descripta por Morgagni en 1761 en estudios anatomopatológicos y, posteriormente, fue diagnosticada en un paciente en vivo por Munker en 1958 mediante la angiografía coronaria.

Describen una dilatación localizada de la luz coronaria de más del $50 \%$ del diámetro respecto de los segmentos adyacentes normales. Su prevalencia es baja, abarca el 0,15-4,9\% ${ }^{1}$ y afecta principalmente al sexo masculino. Se diferencian de las ectasias, que son dilataciones que comprometen al menos el $50 \%$ de la longitud del vaso. Se clasifican según su diámetro (pequeño $<5 \mathrm{~mm}$, mediano entre 5 y $8 \mathrm{~mm}$, y gigante $>8$ $\mathrm{mm}$ ); según su forma (sacular o fusiforme), según su localización, si son verdaderos o pseudoaneurismas ${ }^{3}$

El factor causal más frecuente es la aterosclerosis (90\%), seguida de la enfermedad de Kawasaki, otros desórdenes vasculíticos (arteritis de Takayasu), enfermedades del tejido conectivo, procesos infecciosos, uso de drogas ilícitas y enfermedades congénitas (displasia fibromuscular) ${ }^{4-7}$.

En su patogenia intervienen los mismos factores de riesgo

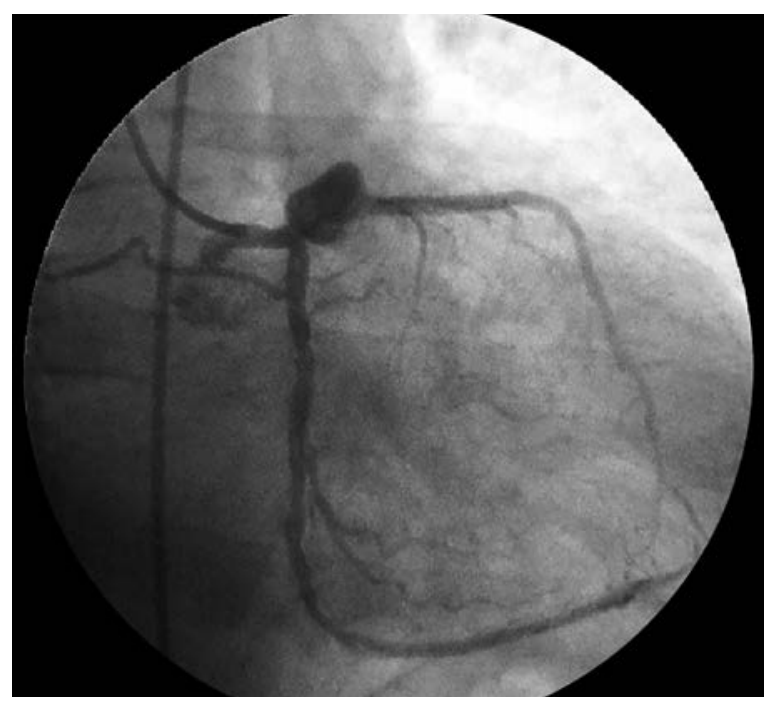

Figura 3.

de la enfermedad coronaria. Exámenes histológicos demostraron hialinización y depósitos lipídicos que alteran y debilitan la íntima y la media, con destrucción de los componentes musculares elásticos y la consecuente dilatación. Se han descripto también algunos genes que podrían asociarse a la formación de aneurismas coronarios y la relación de estos con la implantación de stents. Los mecanismos propuestos para explicar estos casos son la disección residual, la malposición del stent y el efecto de las drogas citotóxicas que retrasan la reendotelización neointimal en el caso de los stents farmacoactivos ${ }^{3,6,8}$.

Clínicamente, su presentación varía desde formas asintomáticas hasta el síndrome coronario agudo y la muerte súbita. Sus potenciales complicaciones son la trombosis y embolización distal, isquemia o infarto de miocardio, disección, vasoespasmo, calcificación, ruptura o fistulización que pueden desencadenar episodios de muerte súbita, síndromes coronarios agudos o taponamiento cardíaco ${ }^{3}$.

Su diagnóstico, en general, es un hallazgo incidental en la cinecoronariografía. La arteria afectada en la mayoría de las veces es la coronaria derecha, seguida por la descendente anterior o la circunfleja (diferentes estudios presentan diferentes incidencias) $)^{5}$. Otros métodos diagnósticos no invasivos incluyen la tomografía, el ecocardiograma y la resonancia magnética.

El manejo terapéutico de esta entidad incluye varias opciones: quirúrgico, percutáneo o médico. Su sola presencia no es indicativa de tratamiento invasivo, que debe reservarse para pacientes con IAM y/o angina recurrente y AAC con alto riesgo de ruptura ( $\mathrm{AAC}$ gigantes o de rápido crecimiento, AAC saculares, síntomas compresivos y en pacientes con enfermedad arteriosclerótica severa que requieren revascularización miocárdica).

Al compartir, en la mayoría de los casos, un mecanismo fisiopatológico similar a la aterosclerosis, la terapia farmacológica consiste en medicación antiplaquetaria, anticoagulantes, estatinas y vasodilatadores, además del control de los factores de riesgo cardiovasculares. Si bien no hay consenso sobre el manejo óptimo de los AAC, las decisiones deben adaptarse a cada paciente en particular, teniendo en cuenta la forma de presentación clínica, la etiología, el tamaño, la localización, su crecimiento con el tiempo y su asociación a enfermedad aterosclerótica. La evolución en el desarrollo de dis- 


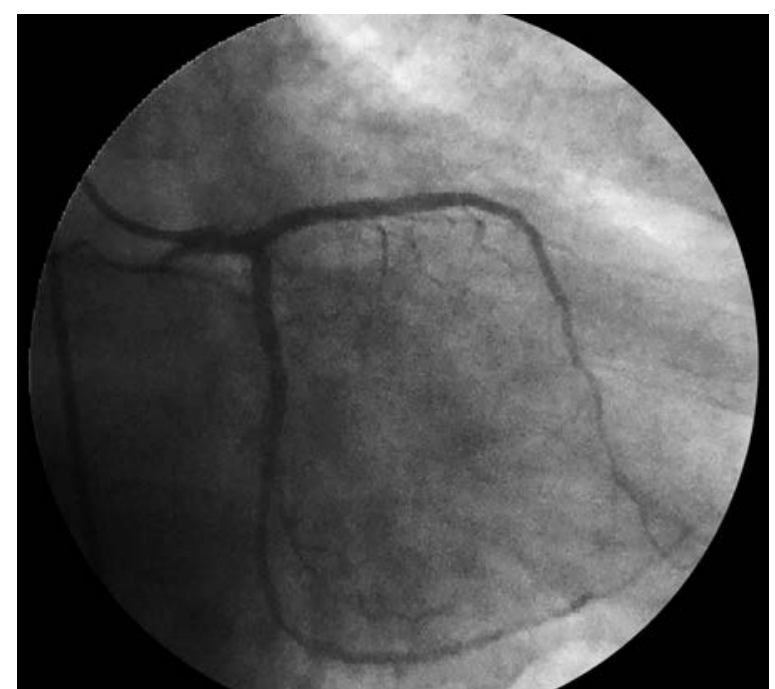

Figura 4.

positivos endovasculares ha hecho que esta sea la principal opción terapeútica en la mayoría de los pacientes, teniendo como primera alternativa (y la que cuenta con mayor canti- dad de casos reportados) los stents coronarios cubiertos con PTFE y dejando en segundo lugar, con mucho menor tasa de uso por la mayor complejidad técnica y mayores chances de complicaciones, la utilización de coils. ${ }^{2}$

Finalmente, la cirugía se recomienda en pacientes que no pueden ser tratados por vía percutánea o en portadores de aneurismas saculares de gran tamaño con alto riesgo de ruptura.

\section{CONCLUSIÓN}

Los aneurismas de las arterias coronarias son entidades poco frecuentes que representan una expresión de la enfermedad coronaria aterosclerótica, en la mayoría de los casos, por lo cual su tratamiento se orienta a la prevención primaria y secundaria de la aterosclerosis. El tratamiento invasivo se limita a algunas situaciones clínicas en particular, siendo el tratamiento endovascular con stents cubiertos con PTFE, la primera opción a considerar.

\section{AGRADECIMIENTOS}

Se agradece al T. R. Claudio Alva por su colaboración durante los procedimientos

\section{BIBLIOGRAFÍA}

1. Características morfológicas de los aneurismas de arterias coronarias. Cir Cardiov. 2014;21(4):252-258. Soc. Española de Cirugía Toracica-cardiovascular.

2. Use of polytetrafluoroethylene-covered stent for treatment of coronary artery aneuriysm. Catheterizaction and cardiovascular interventions 66:203-208. 2005.

3. Aneurisma coronario complicado. Rev Fed Arg Cardiol 2014,43 (3), 155-158.

4. Giant coronary artery aneurysm. Review and update. Texas heart inst J 2014; 41 (6): 603-8)

5. Aneurisma de la arteria coronaria. Cohen P, O'Gara PT. Cardiol Rev. 2008; 16: 301-304 [PubMed]

6. Enfermedad aneurismática coronaria. Acta médica colombiana vol 34. N1. Enero-marzo 2009.

7. Coronary Artery Aneurysm Presenting as Non-STElevation Myocardial Infarction. DOl: 10.7759/cureus. 1436

8. Ozaki K, Tanaka T. Molecular genetics of coronary artery disease. J Hum 9. Genet (2016) 61:71-7. doi:10.1038/jhg.2015.70 